Gastroenterology

\section{Abdominal pain and vomiting in an elderly diabetic woman}

\section{S Koduri, S H Guptha}

Answers on $p 712$.

A 82 year old white woman was admitted with a history of heartburn nausea and vomiting of three days' duration. She also complained of pain in her lower abdomen. She was being treated for type 2 diabetes mellitus, hypertension, and atrial fibrillation. Her medication consisted of metformin, tolbutamide, digoxin, aspirin, and bendrofluazide. She had previously had an ultrasound scan of her abdomen that incidentally showed biliary stones, but there was no previous history of biliary colic. She had no previous history of abdominal surgery. On admission she was nauseated and had bilious vomiting. Her pulse rate was 81 beats/min, irregularly irregular, blood pressure was 150/86 mm Hg, core temperature was raised at $38 \mathrm{C}$, and there was no pallor or icterus. The abdomen was distended with tenderness in left inguinal fossa but there was no guarding or rigidity. The bowel sounds were exaggerated. Rectal examination revealed an empty rectum. The rest of the examination was within normal limits. Investigations showed a normal haemoglobin concentration but raised white cell count with predominant neutrophilia. Renal and liver function tests were within normal limits.

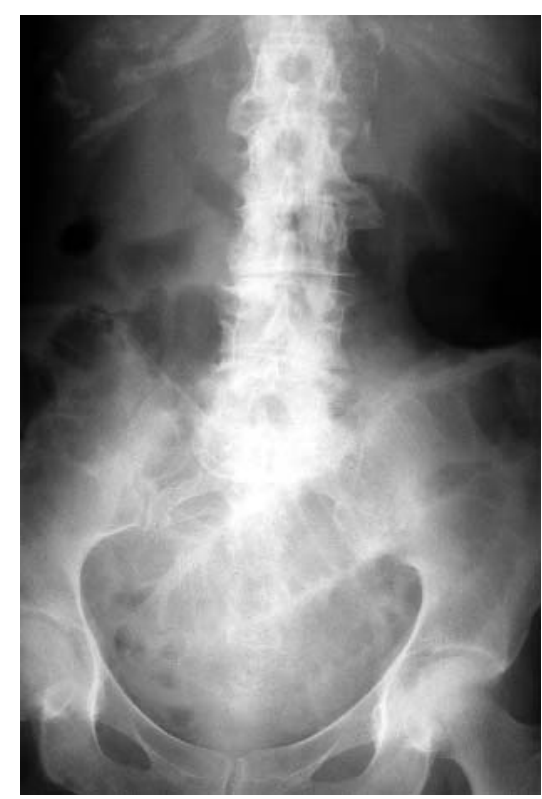

Figure 1 Plain radiograph.

Serum glucose was raised at $16 \mathrm{mmol} / \mathrm{l}$. An electrocardiogram showed atrial fibrillation with a heart rate of 95 beats/min. Her abdominal radiograph is shown below (fig 1). The patient

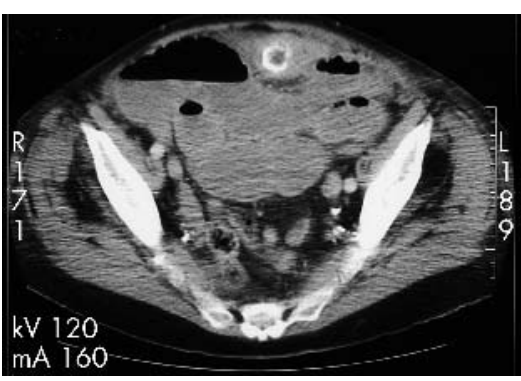

Figure 2 Computed tomogram.

was diagnosed with acute intestinal obstruction and was kept nil by mouth, started on intravenous broad spectrum antibiotics, and a nasogastric tube was placed with free drainage pending computed tomography of the abdomen (fig 2).

\section{QUESTIONS}

(1) What abnormalities are seen on the plain film (fig 1) and computed tomogram (fig 2) of the abdomen?

(2) What is the diagnosis?

(3) What other variants of this disease are known to occur?

Postgrad Med J 2003;79:709.

doi: 10.1136/pgmi.2002.004127q

.................

Authors' affiliations

S Koduri, S H Guptha, Department of Medicine, Hinchingbrooke Hospital, Huntingdon PE29 6NS, UK

Correspondence to: Dr Koduri; sreekanthkoduri@hotmail.com

Submitted 12 December 2002

Accepted 3 January 2003 


\section{Proptosis in an asthmatic patient}

\section{T S Shah, P Sundaram, J D Rege, J M Joshi}

Answers on $p 712$.

$\Lambda$ 33 year old male non-smoking farmer was admitted to the neurosurgery services with a history of diplopia, visual loss, epistaxis, and a left orbital swelling. He was referred to us for preoperative evaluation in view of chest radiographic opacities. He had cough with mucoid sputum, breathlessness for two years, and had been treated with antituberculosis drugs for the same.

On clinical examination he had proptosis of the left eye and respiratory system examination revealed bilateral ronchi and crackles. There were fleeting nodular opacities on serial chest radiographs. His sputum for acid fast bacilli was negative on several occasions. Laboratory examination showed a normal blood count, renal and liver profile. Serum IgE concentration was greater than $1000 \mathrm{IU} / \mathrm{ml}$ (normal values 20-100 IU/ml), cytoplasmic antineutrophil cytoplasmic antibody (C-ANCA) was positive with a titre of 1:20 (positive

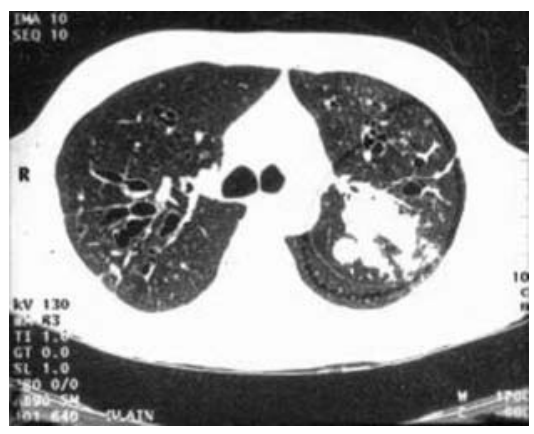

Figure 1 High resolution computed tomogram of the chest showing areas of mucoid impaction with bilateral bronchiectasis.
$>1: 10)$, and specific IgE for Aspergillus fumigatus was positive. Spirometry showed an obstructive abnormality with poor bronchodilator reversibility.

High resolution computed tomography of the chest showed bilateral cystic bronchiectasis with mucoid impaction (fig 1). Magnetic resonance imaging (MRI) of the orbit with sinuses revealed a large mass involving the left ethmoidal and maxillary sinuses (fig 2) with involvement of the floor of the orbit and extension into the medial, lateral recti, and inferior and superior orbital

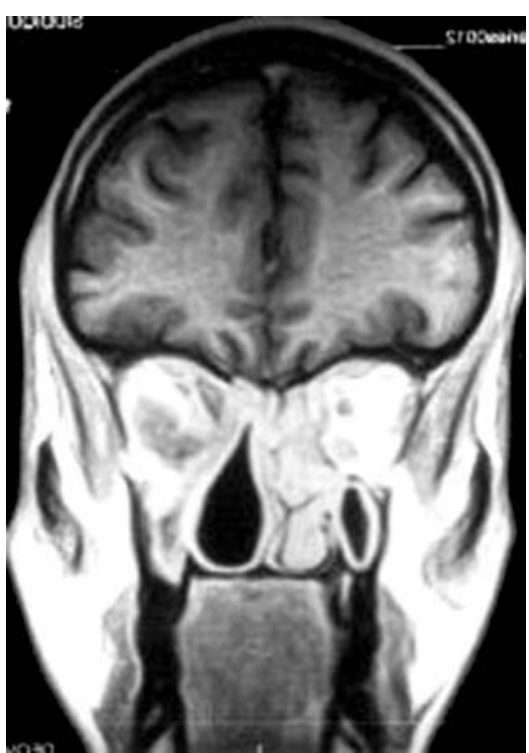

Figure 2 MRI of the orbits and sinuses showing a mass involving the left ethmoidal and maxillary sinuses.

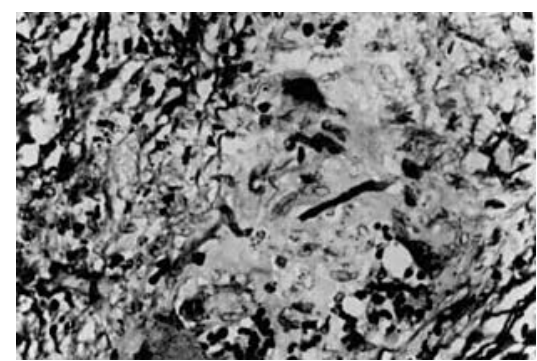

Figure 3 Photomicrograph of the histopathology slide of the operated orbital mass.

fissure. The optic nerve was compressed with adjacent cavernous sinus erosion. The patient was treated with inhaled bronchodilators, oral steroids, and was subjected to surgery for his orbital tumour. Histopathology of the tumour showed a film of collagenous tissue, bits of degenerative bony tissue hyalinisation, inflammation, and giant cells within which were a few filamentous structures with short acute angled hyphae (fig 3). There was no vasculitis.

\section{QUESTIONS}

(1) What is the diagnosis of this respiratory condition?

(2) Which respiratory diseases have coexistent sinus and orbital involvement and what was the cause in this patient? (3) What is the significance of C-ANCA in chronic lower respiratory tract infections?

Postgrad Med J 2003;79:710. doi: $10.1136 /$ pgmi.2002.003095q

\section{Authors' affiliations}

T S Shah, P Sundaram, J D Rege, J M Joshi, Department of Respiratory Medicine, T N Medical College and B Y L Nair Hospital, Mumbai

Correspondence to: Professor J M Joshi, Department of Respiratory Medicine, T N Medical College and B Y L Nair Hospital, Mumbai-400008, India deptrespmed@hathway.com

Submitted 15 November 2002 Accepted 16 January 2002 
Rheumatology

\section{Rheumatoid arthritis and neck pain}

\section{R Thonse, M Belthur}

Answers on $p 713$.

A woman aged 55 years, with a longstanding history of polyarticular rheumatoid arthritis, presented with neck pain. There were no symptoms of neurological involvement. Examination revealed mild tenderness at the upper cervical spine. There were no signs of any neurological deficit. Lateral radiographs of the cervical spine

(in flexion and extension) and magnetic resonance imaging (MRI) of the cervical spine are shown (figs 1 and 2).

\section{QUESTIONS}

(1) What are the features seen on the lateral radiographs of the cervical spine? (2) What abnormalities are seen in the MRI scan?

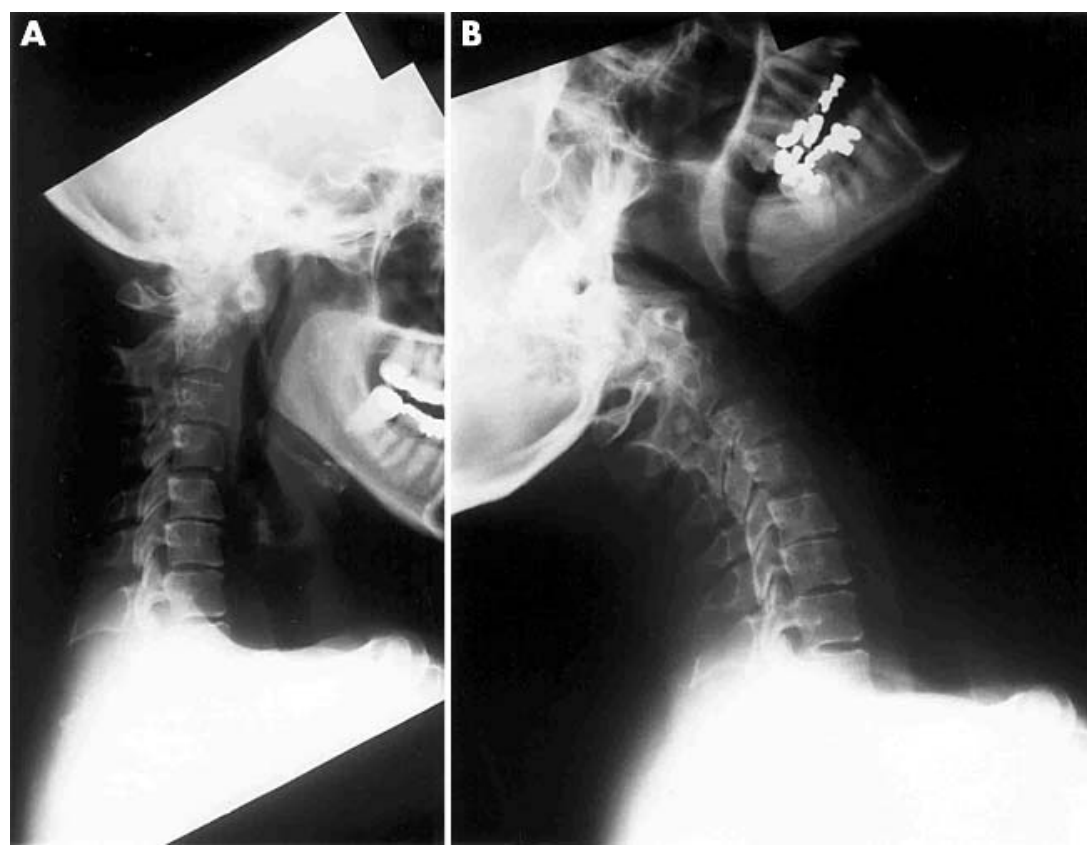

Figure 1 Lateral radiographs of the cervical spine: (A) flexion view and (B) extension view.

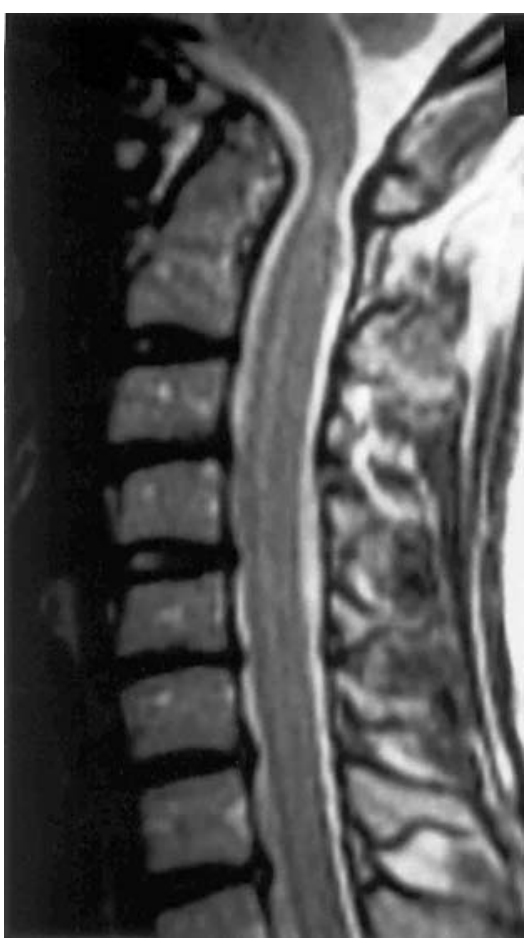

Figure 2 MRI scan of the neck.

(3) What is the likely diagnosis?

(4) How can this condition be treated?

Postgrad Med J 2003;79:711.

doi: 10.1136/pgmi.2002.004275q

\section{Authors' affiliations}

R Thonse, Hull Royal Infirmary, Hull M Belthur, Royal Orthopaedic Hospital, Birmingham

Correspondence to: Dr Thonse; thonser@hotmail.com

Submitted 17 December 2002 Accepted 18 February 2003 